\title{
The Comparative Effectiveness and Safety of Different Anticoagulation Strategies for Treatment of Left Atrial Appendage Thrombus in the Setting of Chronic Anticoagulation for Atrial Fibrillation or Flutter
}

\author{
Karol Kołakowski ${ }^{1}$ - Michał M. Farkowski ${ }^{1}$ Mariusz Pytkowski ${ }^{1}$. Piotr Gardziejczyk ${ }^{1}$ - Ilona Kowalik ${ }^{2}$. \\ Rafał Dąbrowski $^{2} \cdot$ Bohdan Firek $^{2} \cdot \mathrm{Krzysztof} \mathrm{Jaworski}^{2} \cdot$ Anna Klisiewicz $^{3} \cdot$ Aleksander Maciąg $^{1}$
}

Accepted: 6 October 2021 / Published online: 20 October 2021

(C) The Author(s) 2021

\begin{abstract}
Purpose To compare effectiveness of different treatments for atrial fibrillation (AF) patients who were scheduled for cardioversion (CV) or ablation (CA) presenting with left atrium appendage (LAA) thrombus despite chronic oral anticoagulation therapy (OAC).

Methods This was a retrospective cohort study. We analyzed 2014-2019 medical records of patients scheduled for CV or CA of AF who were diagnosed with LAA thrombus despite optimal OAC and had a follow-up transesophageal echocardiogram (TOE). Changes in treatment were divided into the following groups: switch to a drug with different mechanism of action, switch to a drug with similar mechanism of action, initiation of combination therapy, or deliberate no change in treatment. Patients with contraindications to non-vitamin K antagonists were excluded from the analysis.

Results We analyzed data of 129 patients comprising 181 cycles of treatment. The overall effectiveness of LAA thrombus dissolution was $51.9 \%$ regardless of the number of cycles and $42.6 \%$ for the first cycle of treatment. Any change of treatment was more effective than deliberate no change-OR 2.97 [95\% CI: 1.07-8.25], $P=0.031$, but no particular strategy seemed to be more effective than the other. Left atrium area (OR 0.908 [95\% CI: 0.842-0.979]) and number of treatment cycles (OR 0.457 [95\% CI: $0.239-0.872]$ ) were both adversely related to thrombus resolution. There was one ischemic and three bleeding adverse events during the treatment.

Conclusion LAA thrombus resolution in patients already on OAC may require a change of previous OAC treatment but the overall effectiveness of dissolution seems to be about $50 \%$.
\end{abstract}

Keywords Anticoagulation · Atrial fibrillation $\cdot$ Dissolution $\cdot$ Left atrial appendage $\cdot$ Thrombus $\cdot$ Resolution

\section{Introduction}

Atrial fibrillation (AF) is the most common sustained arrhythmia and is associated with fivefold increased occurrence of ischemic stroke mainly due to left atrium appendage (LAA) thrombus formation [1]. To reduce thromboembolic risk, oral anticoagulation therapy (OAC) should be implemented and guided based on the $\mathrm{CHA}_{2} \mathrm{DS}_{2}$-VASc scale [2]. While there is general consensus that rhythm and rate-control strategies are more or less equal when longterm prognosis is considered more recent, data derived from randomized controlled trials or meta-analyses indicate that successful rhythm control specially if instigated earlier in the course of AF might be superior to the standard rate-control 
strategy [2-4]. This effect might be even more pronounced in patients undergoing AF ablation, the most effective rate-control strategy currently available, especially among patients with heart failure [3,5]. Patients scheduled for elective cardioversion $(\mathrm{CV})$ or ablation should be treated with $\mathrm{OAC}$ for at least 3 weeks prior to the procedure regardless of $\mathrm{CHA}_{2} \mathrm{DS}_{2}$-VASc score $[2,6]$. When there is a need to perform a procedure in a patient without prior anticoagulation, a transesophageal echocardiography (TOE) may be used to rule out a LAA thrombus. Many centers tend to do a TOE irrespectively of previous treatment as more and more data shows up describing LAA thrombus or thromboembolic event despite continuous oral anticoagulation [7-9]. Some centers disqualify patients from sinus rhythm restoration only due to LAA sludge; however, there is some evidence that this phenomenon does not link with subsequent neurological disorders [10].

LAA plays an important role in left atrium contraction; produces atrial and brain natriuretic peptides; may be a potential site of origin of AF triggers; and, last but not least, is a major site of origin of cardiac thrombus in patients with stroke [11-13]. LAA thrombus is a well-recognized contraindication to both cardioversion and AF ablation due to elevated risk of stroke or systemic embolization $[2,6]$. At least 3 weeks of successful OAC treatment should precede another imaging study to exclude thrombus and proceed with the procedure. There is no clear advice for patients with thrombus resistant to $\mathrm{OAC}$ and decisions should be taken on a case-by-case basis without strong support in available evidence $[2,6]$. This includes switching to rate-control strategy; cardioversion in the setting of a long-standing, fixed LAA thrombus; or LAA closure despite persistent thrombus.

According to existing evidence, there is overall $0.5-14 \%$ risk of LAA thrombus formation in AF patients and it depends on $\mathrm{CHA}_{2} \mathrm{DS}_{2}$-VASc score, AF type (paroxysmal/ non-paroxysmal) and OAC used-vitamin $\mathrm{K}$ antagonist (VKA) — or novel oral anticoagulant (NOAC) [14, 15]. In a large meta-analysis by DiMinno et al., LAA thrombus was present in 3.4\% (1.3-8.7\%) patients treated with OAC and in $7.4 \%(2.3-21.5 \%)$ OAC-naive patients [16]. Wyrembak et al. performed TOE in AF patients before ablation, and LAA thrombus was found in 1.55\% patients on VKA and in $0.24 \%$ patients on NOAC, whereas Frenkel et al. found LAA thrombus in $2.9 \%$ patients on VKA and in $4.4 \%$ patients on NOAC $[17,18]$. In the "Extra Study," 53 patients with LAA thrombus who were OAC-naive or inadequately treated with VKA underwent rivaroxaban therapy. During a follow-up of 6 weeks, $41.5 \%$ of them had the LAA thrombus dissolved [19]. There is evidence that patients with LAA thrombus hitherto OAC-naive can be treated both by VKA or NOAC [6, 20-22].

Little is known, however, about what kind of action should be undertaken if patient is diagnosed with thrombus in LAA despite apparently correct chronic OAC treatment [23]. Published data is scarce and limited mainly to small case series or case reports [9, 24-26]. In a survey conducted by the European Heart Rhythm Association (EHRA), responders reported over a dozen of potential options when dealing with OAC-resistant thrombus [8]. Recent EHRA NOAC guidelines state that treatment of resistant LAA thrombi should be selected on individual basis: both switching between different NOACs or changes to VKA are admissible [6].

The aim of this study was to assess clinical effectiveness and safety of different treatment strategies of LAA thrombus dissolution in patients already on OAC who are scheduled for $\mathrm{CV}$ or catheter ablation.

\section{Methods}

\section{Study Design}

This was a retrospective cohort study conducted in a tertiary care cardiological center. The study has been approved by Local Bioethics Committee. Hospital medical records covering years 2014-2019 were reviewed to identify patients already on OAC who were diagnosed with LAA thrombus during TOE performed due to elective electrical cardioversion or catheter ablation and had a TOE follow-up visit. The following data was extracted: age, sex, body weight, body mass index (BMI), creatinine clearance, comorbidities, forms of arrhythmia (AF or atrial flutter, AFL), used OAC, change of OAC, echocardioghraphic parameters (including left ventricular ejection fraction, LA diameter and area), duration of treatment, number of cycles of treatment. TOE examinations were performed by experienced, certified physicians in the setting of two independent echocardiography laboratories accredited by the Section of Echocardiography of the Polish Cardiac Society with the highest class "C" (reference units). GE Healthcare Vivid E95 Cardiac Ultrasound and GE Healthcare Vivid E9 Cardiac Ultrasound machines were used. As per standard in our ECHO laboratories, the outcome of LAA examination before the procedure was confirmed by second echocardiographist and disagreements were resolved by discussion. Echocardiographists were not informed about the type of chronic OAC but were aware of the indication for TOE and had full access to patients' medical records if needed. Patients who were treated with inadequate $\mathrm{OAC}$ dose (e.g., rivaroxaban $15 \mathrm{mg}$ without renal impairment) or who omitted at least one dose in past 4 weeks, as well as patients suffering from moderate-tosevere mitral stenosis or with a mechanical valve implanted, were excluded from the analysis. Patients who apart from TOE had additionally another image study performed, e.g., 
computed tomography (CT) showing no thrombus or obvious artifact, were also excluded.

We formed a 3-grade scale to evaluate the size of the encountered thrombi:

\section{1-small, soft thrombus}

2-solid thrombus occupying less than one-half of the atrial appendage 3-solid thrombus occupying more than one-half of the atrial appendage

Examples of thrombus and sludge are shown in Figs. 1, 2, and 3. Unequivocal "sludge" (Fig. 3) was treated in our study as LAA free of thrombus. The procedures were performed as scheduled, and therefore, those patients were not included in this analysis. However, thick sludge on the verge
Fig. 1 Thrombus grade 3 occupying almost all volume of left atrium appendage
Fig. 2 Thrombus grade 2 occupying nearly half of left atrium appendage
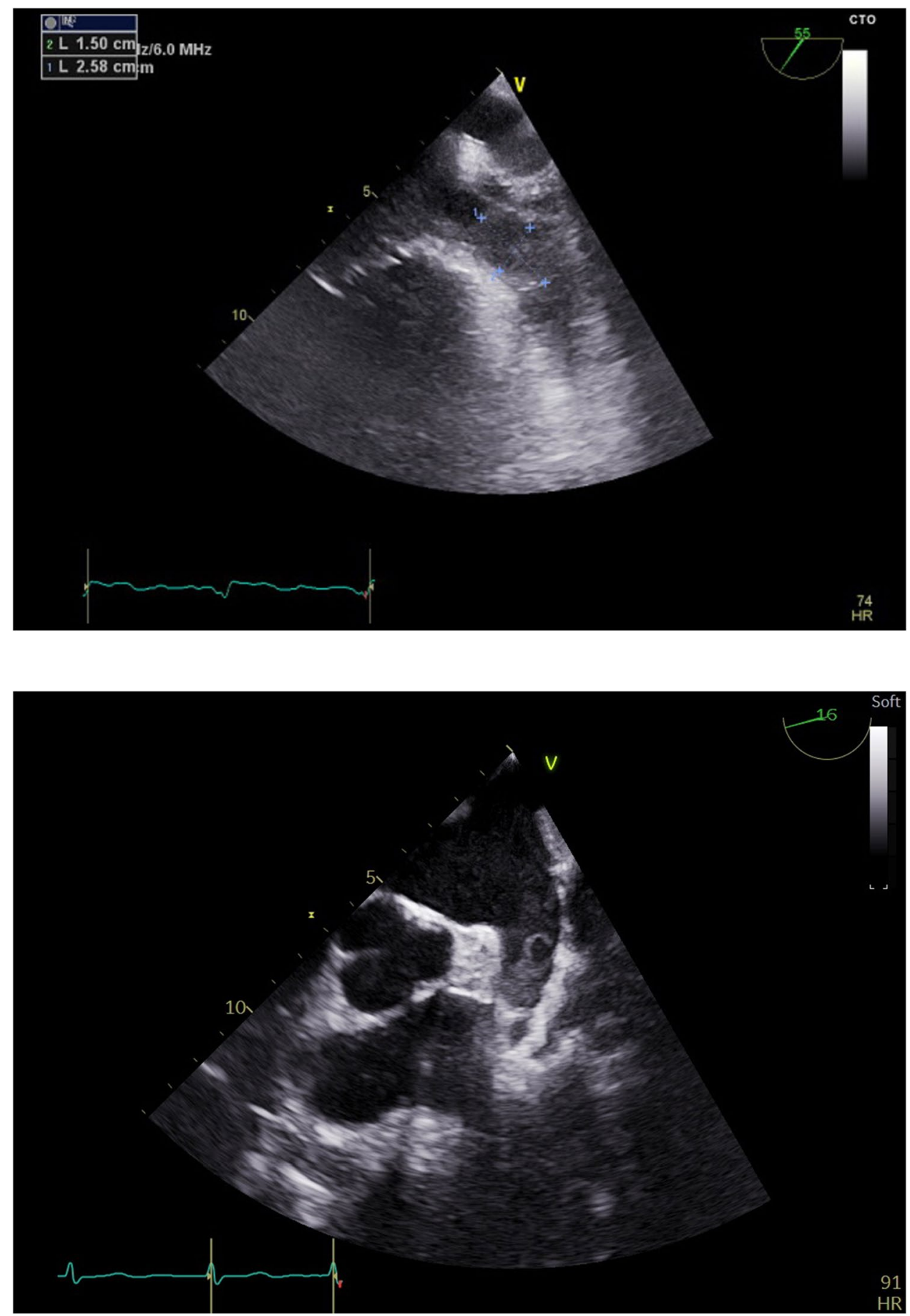
Fig. 3 Unequivocal "sludge" in left atrium appendage

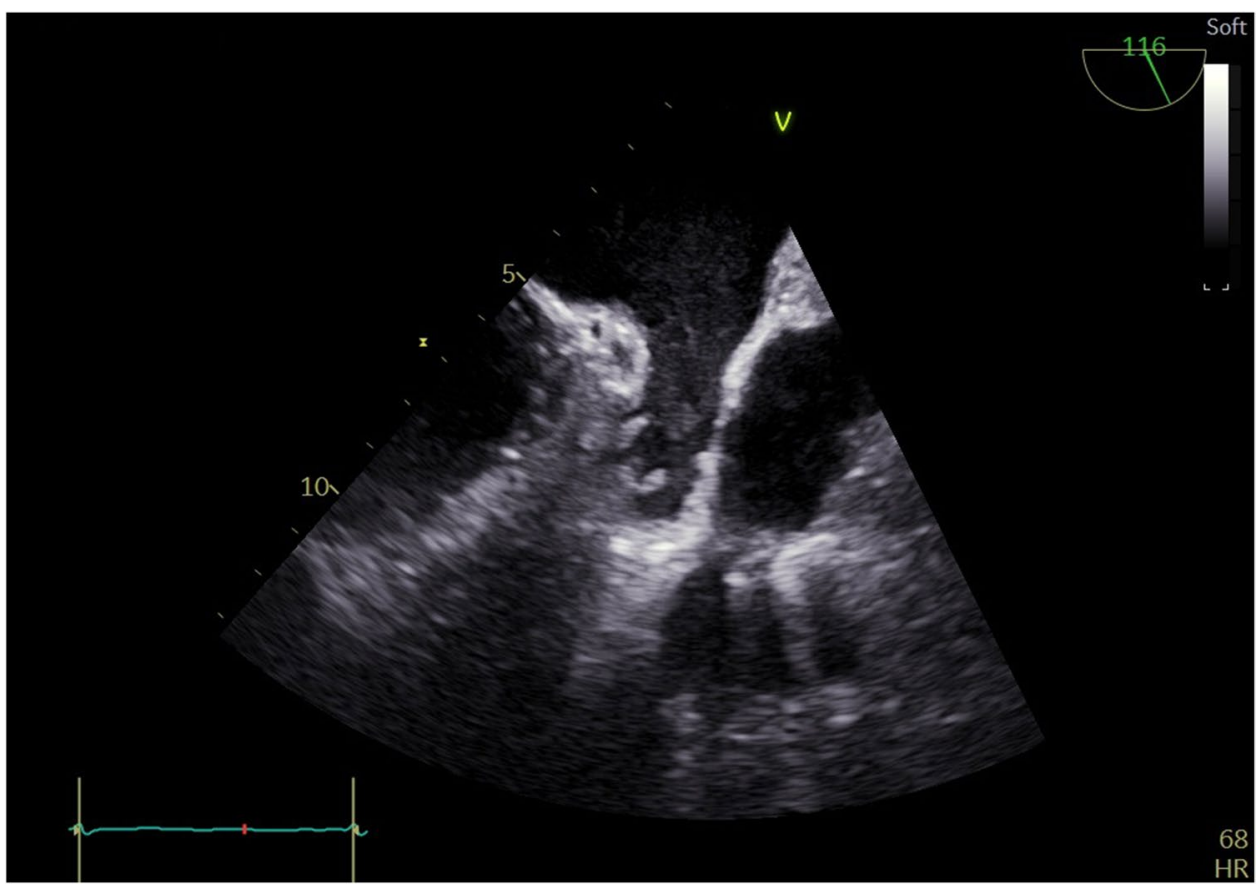

of thrombus formation indistinguishable from soft thrombus by the study echocardiographers was considered grade 1 thrombus.

Patients who had been treated for at least 3 weeks and on follow-up TOE were again diagnosed with LAA thrombus being still in rhythm control strategy, and were scheduled for another TOE and a second, third etc. cycle of treatment. Our main analysis comprises all cycles of treatment and each cycle is treated as an independent case. Based on clinical experience and published data, we identified the following possible changes in OAC treatment strategy.

1- switch to drug of different mechanism of action (e.g., $\mathrm{VKA} \rightarrow \mathrm{NOAC}, \mathrm{NOAC} \rightarrow \mathrm{VKA}, \mathrm{NOAC} \rightarrow$ low molecular weight heparin (LMWH)),

2 - switch to drug of similar mechanism of action (e.g., $\mathrm{NOAC} \rightarrow \mathrm{NOAC}, \mathrm{VKA} \rightarrow \mathrm{VKA}$ including also dose increase),

3-implementation of combination therapy:

a-by adding antiplatelet therapy (APT) (e.g., $\mathrm{VKA} \rightarrow \mathrm{VKA}+\mathrm{APT})$

$\mathrm{b}$ - by switching antithrombotic drug and adding APT (e.g., NOAC $\rightarrow$ VKA + APT)

c-by adding second antithrombotic drug (e.g., $\mathrm{VKA} \rightarrow \mathrm{VKA}+\mathrm{LMWH})$

4-deliberate no change in treatment.

All clinical decisions concerning patients included in this analysis: initial drug treatment, change and type of OAC, decision on continuation of rhythm control strategy, were undertaken independently by physicians on site at the time of hospitalization and therefore reflected actual clinical practice in different departments of study center.

\section{Statistical Analysis}

Univariable analysis was applied to both continuous and categorical variables. Continuous variables are reported as means SD or as median and interquartile range. Student's $t$-test was performed for comparison of normally distributed continuous data, and Mann-Whitney test for comparison of non-normally distributed continuous data. Categorical variables are presented as counts and percentages. Amonggroup comparisons $2 \times 2$ were made using a chi-square test of independence or Fisher's exact test if any expected cell count was less than 5. Ordinal variable was compared using Cochran-Mantel-Haenszel statistics.

Penalized likelihood logistic regression was used to estimate the odds ratios for patients in whom the therapy was effective vs. ineffective. Odds ratios were calculated for demographic and other baseline characteristics and medications. A stepwise multivariable logistic regression analysis was performed to establish the relationship between patients' characteristics and efficacy of thrombus resolution, including into the model of all the candidate variables. A significance level of 0.05 was required for a variable to enter and stay in the model. Hosmer and Lemeshow goodness-of-fit test and percent concordant were calculated.

A two-sided $p<0.05$ was considered statistically significant. All analyses were performed using SAS statistical software version 9.4 (SAS Institute, Inc., Cary, NC, USA). 


\section{Results}

We reviewed 8028 TOEs to initially identify 161 patients who had a LAA thrombus despite chronic OAC treatment and had a follow-up TOE. We excluded from the analysis 16 patients due to contraindications to NOAC, 11 patients due to inadequate or unclear treatment, and another 5 patients because other imaging studies which ruled out thrombus and CV/ablation were performed as scheduled. Thus, 129 patients were enrolled: 102 with $\mathrm{AF}$ and 27 with atrial flutter (AFL). The patients' selection process is presented in Fig. 4.

Each TOE was performed on the day of the scheduled procedure or the day before it. The follow-up TOE was performed after a median of 62 days (IQR: 42-99 days, range 22-182 days) since the change in antithrombotic treatment. Among enrolled patients, 94 had one change of treatment and 35 had more than one change of treatment comprising a total of 181 cycles of treatment.

\section{Baseline Characteristics}

Table 1 summarizes the initial antithrombotic therapy. Rivaroxaban was administered 15 mg q.d. in 8 cases-in 6 cases due to renal impairment with eGFR $<50 \mathrm{ml} / \mathrm{min}$, in one case due to serious bleeding on adequate dose, and in one case due to concomitant antiplatelet therapy and high
Table 1 Anticoagulation therapy before transesophageal echocardiography (TOE)

\begin{tabular}{ll}
\hline Anticoagulation therapy & $N=129$ \\
\hline VKA & $53(41.1 \%)$ \\
Warfarin & $17(13.2 \%)$ \\
Acenocoumarol & $36(27.9 \%)$ \\
NOAC & $74(57.4)$ \\
Rivaroxaban & $50(38.8 \%)$ \\
Dabigatran & $19(14.7 \%)$ \\
Apixaban & $5(3.9 \%)$ \\
LMWH & $2(1.5 \%)$ \\
APT* & $8(6.2 \%)$ \\
ASA & $5(3.9 \%)$ \\
Clopidogrel & $5(3.9 \%)$ \\
\hline
\end{tabular}

$A P T$, antiplatelet therapy; $A S A$, acetylsalicylic acid; $L M W H$, low molecular weight heparin; $N O A C$, novel oral anticoagulant; $V K A$, vitamin $\mathrm{K}$ antagonist

*Additional treatment to oral anticoagulation

bleeding risk. Dabigatran was administered $110 \mathrm{mg}$ b.i.d. in 5 patients - one of them due to concomitant dual antiplatelet therapy and high bleeding risk because of recent PCI with DES implantation, one-due to serious bleeding when on adequate dose, one-because of age $>80$ years, and twobecause of renal impairment. Apixaban was administered $2.5 \mathrm{mg}$ b.i.d. in one case due to history of serious bleeding
Fig. 4 Flow diagram of patients' selection for the analysis. AF, atrial fibrillation; AFL, with atrial flutter; OAC, oral anticoagulant; NOAC, novel oral anticoagulant; TOE, transesophageal echocardiography

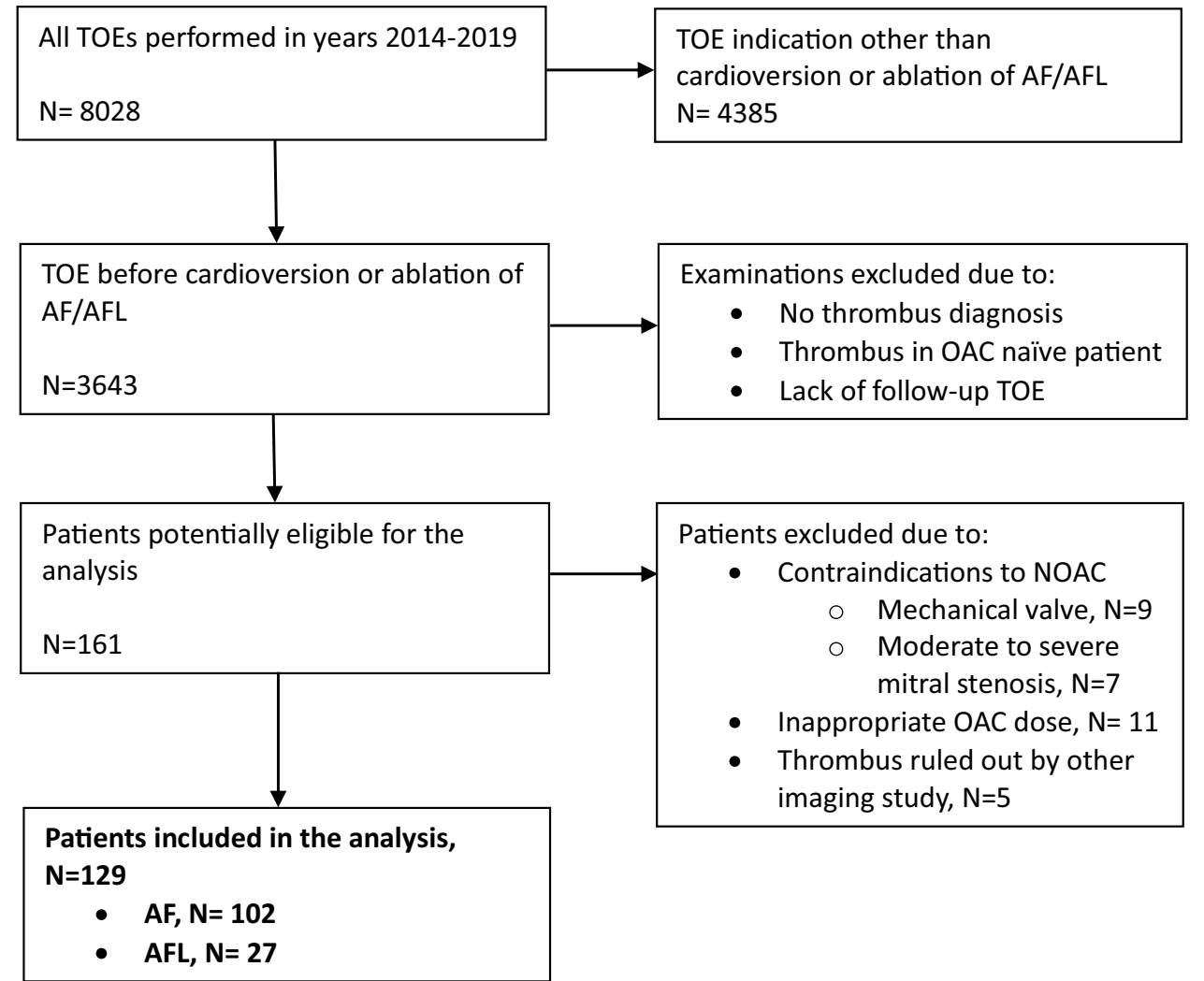


on adequate dose. APT was an additional treatment in 9 patients: 4 patients were taking aspirin and another 4 clopidogrel, whereas one patient was on dual APT due to recent percutaneous coronary intervention.

Baseline characteristics of patients enrolled in our study and univariate analysis are presented in Table 2. Overall, 67 patients $(51.9 \%)$ succeeded in dissolving the thrombus regardless of the number of treatment cycles. Left atrium area and number of cycles were adversely related to LAA thrombus resolution, whereas hypertension was a positive prognostic factor of thrombus resolution. The overall effectiveness of the first change of treatment was $42.6 \%$.

On multivariate analysis, two variables were independent predictors of thrombus resolution failure: left atrium area (OR 0.908 [95\% CI: 0.842-0.979]; $p=0.012$ ) and number of cycles (OR 0.457 [95\% CI: 0.239-0.872]; $p=0.017$ ).

\section{Change of Treatment}

Table 3 summarizes the effectiveness of different strategies of LAA thrombus resolution in all 181 cycles. Any change in the OAC treatment was superior to the strategy where no change in OAC treatment was made-OR 95\% CI: 2.97 [1.07; 8.25], $P=0.031$. Unfortunately, no particular change in OAC treatment was shown to be significantly superior to any other. An additional analysis performed exclusively for the first-cycle change yielded similar results (Supplementary Material, Table S1). General information about OAC treatment in both analyses are presented in Supplementary Material (Table S2 for all cycles of treatment and Table S3 for the first cycle only).

Table 2 Baseline characteristics of patients enrolled to the study, univariate analysis

\begin{tabular}{|c|c|c|c|c|c|}
\hline & $N=129$ & $\begin{array}{l}\text { Successful throm- } \\
\text { bus resolution } \\
N=67(51.9 \%)\end{array}$ & $\begin{array}{l}\text { Unsuccessful } \\
\text { thrombus resolution } \\
N=62(48.1 \%)\end{array}$ & $P$ & Odds ratio $[95 \% \mathrm{CI}]$ \\
\hline Age (years) & $64.4 \pm 11.0$ & $63.8 \pm 11.7$ & $65.2 \pm 10.0$ & 0.466 & $0.988[0.957-1.020]$ \\
\hline Female, $n(\%)$ & $51(39.5 \%)$ & $25(37.3 \%)$ & $26(41.9 \%)$ & 0.592 & $0.824[0.407-1.671]$ \\
\hline Body weight (kg) & $87.1 \pm 19.1$ & $89.4 \pm 20.9$ & $84.6 \pm 16.8$ & 0.195 & $1.014[0.993-1.034]$ \\
\hline BMI $\left(\mathrm{kg} / \mathrm{m}^{2}\right)$ & $30.4 \pm 5.0$ & $31.1 \pm 5.6$ & $29.7 \pm 4.2$ & 0.203 & $1.057[0.970-1.152]$ \\
\hline Creatinine (mg/dl) & $1.05[0.92-1.30]$ & $1.10[1.00-1.30]$ & $1.00[0.90-1.30]$ & 0.351 & $1.104[0.119-1.275]$ \\
\hline EGFR (ml/min) & $61.3 \pm 16.7$ & $61.0 \pm 16.3$ & $61.5 \pm 17.2$ & 0.872 & $0.998[0.978-1.019]$ \\
\hline Structural heart disease*, $n(\%)$ & $90(69.8)$ & $44(65.7)$ & $46(74.2)$ & 0.292 & $0.665[0.311-1.423]$ \\
\hline $\mathrm{HCM}, n(\%)$ & $23(17.8)$ & $9(13.4)$ & $14(22.6)$ & 0.175 & $0.532[0.212-1.336]$ \\
\hline $\mathrm{DCM}, n(\%)$ & $18(13.9)$ & $9(13.4)$ & $9(14.5)$ & 0.859 & $0.914[0.337-2.474]$ \\
\hline Autoimmune diseases, $n(\%)$ & $7(5.4)$ & $3(4.5)$ & $4(6.4)$ & 0.621 & $0.680[0.146-3.166]$ \\
\hline Heart failure, $n(\%)$ & $96(74.4)$ & $51(76.1)$ & $45(72.6)$ & 0.645 & $1.204[0.546-2.658]$ \\
\hline Hypertension, $n(\%)$ & $86(66.7)$ & $51(76.1)$ & $35(56.4)$ & 0.018 & $2.459[1.158-5.223]$ \\
\hline Diabetes mellitus, $n(\%)$ & $42(32.6)$ & $23(34.3)$ & $19(30.6)$ & 0.656 & $1.183[0.565-2.477]$ \\
\hline Vascular disease, $n(\%)$ & $52(40.3)$ & $26(38.8)$ & $26(41.9)$ & 0.717 & $0.878[0.434-1.776]$ \\
\hline History of cancer, $n(\%)$ & $15(11.6)$ & $7(10.4)$ & $8(12.9)$ & 0.664 & $0.787[0.268-2.316]$ \\
\hline Renal impairment, $n(\%)$ & $56(43.1)$ & $29(43.3)$ & $27(43.5)$ & 0.976 & $0.989[0.493-1.986]$ \\
\hline Severe renal impairment (eGFR <30), $n(\%)$ & $4(3.1)$ & $1(1.5)$ & $3(4.8)$ & 0.354 & \\
\hline $\mathrm{CHA}_{2} \mathrm{DS}_{2}$-VASc score & $3.53 \pm 1.63$ & $3.58 \pm 1.57$ & $3.47 \pm 1.72$ & 0.693 & $1.044[0.844-1.291]$ \\
\hline $\operatorname{LVEF}(\%)$ & $43.9 \pm 16.6$ & $44.5 \pm 15.6$ & $43.3 \pm 17.7$ & 0.680 & $1.005[0.983-1.036]$ \\
\hline LA diameter (mm) & $49.1 \pm 6.1$ & $48.6 \pm 6.4$ & $49.6 \pm 5.7$ & 0.402 & $0.974[0.916-1.035]$ \\
\hline LA-area $\left(\mathrm{cm}^{2}\right)$ & $31.0 \pm 5.6$ & $30.0 \pm 5.3$ & $32.1 \pm 5.7$ & 0.032 & $0.929[0.868-0.995]$ \\
\hline Median duration of treatment cycle (days) & $62.0[42-99]$ & $54[40-85]$ & $70.5[43-125]$ & 0.084 & $0.994[0.988-1.000]$ \\
\hline Cycles of treatment & $\begin{array}{l}1.4 \pm 0.8 \\
1 \text { min: } 1, \max : 6\end{array}$ & $\begin{array}{l}1.21 \pm 0.48 \\
1 \min : 1, \max : 3\end{array}$ & $\begin{array}{l}1.61 \pm 1.0 \\
1 \text { min: } 1, \max : 6\end{array}$ & 0.009 & $0.458[0.256-0.818]$ \\
\hline Thrombus size 1 & $67(51.9 \%)$ & $39(58.2 \%)$ & $28(44.4 \%)$ & 0.284 & \\
\hline Thrombus size 2 & $58(45.0 \%)$ & $26(38.8 \%)$ & $32(51.6 \%)$ & & \\
\hline Thrombus size 3 & $4(3.1 \%)$ & $2(3.6 \%)$ & $2(3.2 \%)$ & & \\
\hline
\end{tabular}

Values expressed as mean \pm SD or median $[\mathrm{IQR}]$

$B M I$, body mass index; $D C M$, dilated cardiomyopathy; $e G F R$, glomerular filtration rate; $H C M$, hypertrophic cardiomyopathy; $L A$, left atrium; $L V E F$, left ventricle ejection fraction

*Structural heart disease: ischemic, non-ischemic, or valvular cardiomyopathy 
Table 3 Effectiveness of different strategies of LAA thrombus resolution in 129 patients (all 181 cycles)

\begin{tabular}{lll}
\hline & $\begin{array}{l}\text { Cycles of treatment } \\
N=181\end{array}$ & Efficacy \\
\hline Switch to different mechanism (1) & $69(38.1 \%)$ & $28(40.6 \%)$ \\
Switch to similar mechanism (2) & $38(21.0 \%)$ & $15(39.5 \%)$ \\
Implementation of combination therapy (3) & $47(26.0 \%)$ & $19(40.4 \%)$ \\
- Adding APT (3a) & $27(14.9 \%)$ & $13(48.1 \%)$ \\
- Switch of thrombotic drug and adding APT (3b) & $15(8.3 \%)$ & $5(33.3 \%)$ \\
- Adding second antithrombotic drug (3c) & $5(2.8 \%)$ & $1(20 \%)$ \\
Deliberate no change in treatment (4) & $27(14.9 \%)$ & $5(18.5 \%)$ \\
$1+2+3$ vs. 4; OR 2.97 [95\% CI: 1.07; 8.25]; $\boldsymbol{P}=0.031$ & & \\
\hline
\end{tabular}

$A P T$, antiplatelet therapy; $L A A$, left atrial appendage

$P=0.254$ for all 6 subgroups $(1,2,3 \mathrm{a}, 3 \mathrm{~b}, 3 \mathrm{c}, 4)$

\section{Safety}

In general, antithrombotic therapy used in our analysis was safe and only a few complications were reported. There was one ischemic stroke in a patient treated concomitantly with acenocoumarol (INR goal 2.5-3.5) and aspirin due to a resistant LAA thrombus grade 3 . There were three bleeding events: one bleeding to an eye- on dabigatran $150 \mathrm{mg}$ b.i.d.; one event of hemoptysis-on concomitant acenocoumarol (INR goal 2.5-3.5) and aspirin; one abdominal wall hematoma in a patient treated with LMWH $80 \mathrm{mg}$ b.i.d. None of above patients required blood transfusion or surgical intervention. Additionally, one patient on LMWH suffered from skin allergy and one patient on dabigatran $150 \mathrm{mg}$ b.i.d. reported abdominal pain.

\section{Discussion}

The main findings of this large study over AF patients with LAA thrombus despite adequate antithrombotic treatment prior sinus rhythm restoration are (1) overall effectiveness of LAA thrombus resolution regardless of the adopted strategy and number of cycles was $51.9 \%$, (2) the first-cycle effectiveness of LAA thrombus resolution regardless of the adopted strategy was $42.6 \%$, (3) thrombus resistance was associated with larger number of treatment cycles and wider left atrium area, (4) any change in treatment was three times more effective for thrombus resolution than deliberate no change in treatment, and (5) there was no single most effective strategy for thrombus resolution among different active treatments analyzed in this study.

There are a lot of papers dedicated to risk of LAA thrombus in AF patients and its correlation with prior anticoagulation, $\mathrm{CHA}_{2} \mathrm{DS}_{2}$-VASc score, type of $\mathrm{AF}$ (paroxysmal/ non-paroxysmal), or other clinical factors. Di Minno et al. conducted a meta-analysis which included over twenty thousand patients and showed that LAA thrombus was present in $3.4 \%$ anticoagulated patients and in $7.4 \%$ anticoagulation-naive patients [16]. Zylla et al. showed prevalence of LAA thrombus on particular medication: phenprocoumon (17.4\%), dabigatran (3.8\%), rivaroxaban $(4.1 \%)$ [15]. Trial conducted by Wyrembak et al. showed superiority of NOAC vs. VKA (0.24\% reported LAA thrombi vs. $1.55 \%$, respectively), whereas Frenkel et al. showed that prevalence of LAA thrombus on NOAC and VKA was comparable $[17,18]$. When it comes to clinical factors, apart from $\mathrm{CHA}_{2} \mathrm{DS}_{2}$-VASc scale, chronic kidney disease was related to the higher prevalence of LAA thrombus in the study of Kaplon-Cieslicka et al., while Zhou et al. proved that red cell distribution width and NT-proBNP level would be other risk factors of LAA thrombus formation [27, 28].

Efficacy of LAA thrombus resolution treatment methods in OAC-naive patients was investigated in several studies. In a paper by Niku et al., 127 OAC-naive patients were prescribed adequate anticoagulation, and in $60 \%$ of them, the thrombus was dissolved [29]. In a prospective, multi-center study, Lip GY et al. showed a thrombus resolution rate of $41.5 \%$ (22 of 53) on rivaroxaban treatment [19]. Patients were not anticoagulated (75\%) prior enrollment or were inadequately treated with VKA (25\%).

Data on proper treatment in case of a LAA thrombus diagnosis despite prior anticoagulation and why such phenomenon takes place is scarce. Compliance to treatment may be one of the most important factors but plasma drug concentration was not measured either in our study or in other publications. In some case reports describing resistant thrombi, soluble fibrin and D-dimer concentrations were assessed before and after change of treatment but results were inconclusive [20,26]. According to 2020 ESC guidelines for the diagnosis and treatment of AF when LAA thrombus is found on TOE, a repeat TOE is recommended after $>3$ weeks of antithrombotic treatment but the drug selection is not specified and should be decided on individual basis [2]. In case of our analysis, the duration of treatment cycle was not a significant factor influencing the 
thrombus resolution (Table 2). There are only case reports or analysis of small case series. The biggest group hitherto collected was in the RIVA-TWICE study, and it included 15 patients treated ineffectively with rivaroxaban $20 \mathrm{mg}$ who were then prescribed rivaroxaban $15 \mathrm{mg}$ b.i.d, and in 7 of them, the thrombus was dissolved [25]. Another observation was conducted by Mitamura et al., and it consisted of 6 patients treated initially with dabigatran [24]. After change of treatment (increased dose or switch to warfarin) in 5 of them, the thrombus was dissolved. The LAA thrombus resolution rates achieved in mentioned studies are similar to our findings. Contrary to our expectations, despite markedly larger patient group and more drug combinations investigated than in previously published studies, we were not able to indicate one optimal treatment for our patients. Switching between drugs with different mechanisms of action did not result in clearly better outcomes than other tested options (Table 3). Effectiveness of a combination of OAC and antiplatelet treatment was promising but far from being statistically significant. A combination of two antithrombotic treatments of different mechanism of action, not described before in this setting, was also ineffective.

When assessing NOAC effectiveness, one has to bear in mind potential plasma level fluctuations due to drug-todrug interactions derived from the competitive inhibition of P-glycoprotein (P-gp) transporter or cytochrome P (CYP) induction/inhibition [2, 6, 30, 31]. Especially dronedarone, a potent P-gp and CYP3A4 inhibitor, may increase dabigatran or rivaroxaban plasma concentration to potentially harmful levels. Other drugs posing similar issues to all NOACs are HIV protease inhibitors and fungostatics. As this was a retrospective analysis of medical records, we did not perform any drug plasma level assays. Still, we are not aware of any of those treatments to be prescribed in patients included in our analysis.

As far as safety of therapy is concerned, in our study, no serious bleeding requiring blood transfusion was reported even in patients who had been prescribed off-label treatment comprising two antithrombotic agents. However, there was one event of ischemic stroke on acenocoumarol with ASA in a patient with resistant thrombus. As most of the treatments adopted in our study were more or less according to standard OAC treatment or OAC + antiplatelet drug strategies for which data on safety are widely available [2, 6, 32].

\section{Limitations}

This is a retrospective cohort study with all inherent limitations of this methodology: more of less pronounced selection bias, lack of potentially interesting data, etc. Many variables: type of OAC, duration of follow-up, choice of treatment strategy, were representation of routine clinical practice and did not always follow clinical guidelines to the core. We reported a wide variety of OAC treatments, and apparent overrepresentation of different drugs at baseline might have been observed rather due to their market share than apparent lower clinical effectiveness. In order to lower the heterogeneity of the studied population, we excluded patients with mechanical valves and those with moderateto-severe mitral stenosis-well-recognized contraindications to NOAC. Although our study comprises the largest group of patients with OAC-resistant thrombi hitherto published, still the group might have been too small to demonstrate significant relevance in some comparisons. To exclude any recall bias, all data in the study were extracted from hospital electronical medical records, but in a few patients, we lacked information about LVEF or BMI. We found no information on any previously diagnosed thrombophilia in medical records but such conditions cannot be entirely ruled out in enrolled patients as no formal diagnostic assessment was conducted during hospitalizations.

\section{Conclusion}

The overall effectiveness of left atrial appendage thrombus resolution was $51.9 \%$. Any change of treatment seemed to be superior to no change in previous oral anticoagulant treatment, but no anticoagulant strategy was shown to be more effective than other. Previous failure of thrombus resolution and wider left atrial area were adversely related to oral anticoagulant therapy effectiveness.

Supplementary Information The online version contains supplementary material available at https://doi.org/10.1007/s10557-021-07278-9.

Author Contribution Concept and design of the study: KK, MMF, AM; data extraction and preparation of the database: KK, MMF; statistical analysis: IK; data interpretation and critical revision of the manuscript: all authors.

Funding The analysis was financed by departmental resources of the National Institute of Cardiology, Warsaw, Poland.

Availability of Data and Material The National Institute of Cardiology, Warsaw, Poland, is the sole owner of the analyzed data.

Code Availability Not applicable.

\section{Declarations}

Ethics Approval Although this was a retrospective review of the medial records, we obtained the approval of the Local Bioethics Committee (IK-NPIA-0021-105/1819/19).

Consent to Participate Not required due to retrospective methodology.

Consent for Publication Not required. 
Conflict of Interest MMF received lecturer fees from Pfizer and Boehringer Ingelheim, and RD received lecturer fees from Bayer and Boehringer Ingelheim. All the other authors declare no competing interests.

Open Access This article is licensed under a Creative Commons Attribution 4.0 International License, which permits use, sharing, adaptation, distribution and reproduction in any medium or format, as long as you give appropriate credit to the original author(s) and the source, provide a link to the Creative Commons licence, and indicate if changes were made. The images or other third party material in this article are included in the article's Creative Commons licence, unless indicated otherwise in a credit line to the material. If material is not included in the article's Creative Commons licence and your intended use is not permitted by statutory regulation or exceeds the permitted use, you will need to obtain permission directly from the copyright holder. To view a copy of this licence, visit http://creativecommons.org/licenses/by/4.0/.

\section{References}

1. Wolf PA, Abbott RD, Kannel WB. Atrial fibrillation as an independent risk factor for stroke: the Framingham Study. Stroke. 1991;22(8):983-8.

2. Hindricks G, Potpara T, Dagres N, Arbelo E, Bax JJ, Blomström-Lundqvist C, et al. 2020 ESC Guidelines for the diagnosis and management of atrial fibrillation developed in collaboration with the European Association for Cardio-Thoracic Surgery (EACTS). Eur Heart J. 2021;42(5):373-498

3. Barra S, Baran J, Narayanan K, Boveda S, Fynn S, Heck P, et al. Association of catheter ablation for atrial fibrillation with mortality and stroke: a systematic review and meta-analysis. Int J Cardiol. 2018;266:136-42.

4. Kirchhof P, Camm AJ, Goette A, Brandes A, Eckardt L, Elvan A, et al. Early rhythm-control therapy in patients with atrial fibrillation. N Engl J Med. 2020;383(14):1305-16.

5. Ruzieh M, Foy AJ, Aboujamous NM, Moroi MK, Naccarelli GV, Ghahramani M, et al. Meta-analysis of atrial fibrillation ablation in patients with systolic heart failure. Cardiovasc Ther. 2019;2019:8181657.

6. Steffel J, Collins R, Antz M, Cornu P, Desteghe L, Haeusler KG, et al. European Heart Rhythm Association Practical Guide on the use of non-vitamin $\mathrm{K}$ antagonist oral anticoagulants in patients with atrial fibrillation. Europace. 2021;2021.

7. Calkins H, Hindricks G, Cappato R, Kim YH, Saad EB, Aguinaga L, et al. 2017 HRS/EHRA/ECAS/APHRS/SOLAECE expert consensus statement on catheter and surgical ablation of atrial fibrillation: executive summary. Europace. 2018;20(1):157-208.

8. Farkowski MM, Jubele K, Marín F, Gandjbakhch E, Ptaszynski $\mathrm{P}$, Merino JL, et al. Diagnosis and management of left atrial appendage thrombus in patients with atrial fibrillation undergoing cardioversion or percutaneous left atrial procedures: results of the European Heart Rhythm Association survey. Europace. 2020;22(1):162-9.

9. Miwa Y, Minamishima T, Sato T, Sakata K, Yoshino H, Soejima K. Resolution of a warfarin and dabigatran-resistant left atrial appendage thrombus with apixaban. J Arrhythm. 2016;32(3):233-5.

10. Squara F, Bres M, Scarlatti D, Moceri P, Ferrari E. Clinical outcomes after AF cardioversion in patients presenting left atrial sludge in trans-esophageal echocardiography. J Interv Card Electrophysiol. 2020;57(1):149-56.
11. Beigel R, Wunderlich NC, Ho SY, Arsanjani R, Siegel RJ. The left atrial appendage: anatomy, function, and noninvasive evaluation. JACC Cardiovasc Imaging. 2014;7(12):1251-65.

12. Ghaffarpasand E, Tehrani MD, Marszalek J, Chi G. Non-vitamin $\mathrm{K}$ antagonist oral anticoagulants for the treatment of intracardiac thrombosis. J Thromb Thrombolysis. 2018;46(3):332-8.

13. Murtaza G, Yarlagadda B, Akella K, Della Rocca DG, Gopinathannair R, Natale A, et al. Role of the left atrial appendage in systemic homeostasis, arrhythmogenesis, and beyond. Card Electrophysiol Clin. 2020;12(1):21-8.

14. Kawabata M, Goya M, Sasaki T, Maeda S, Shirai Y, Nishimura $\mathrm{T}$, et al. Left atrial appendage thrombi formation in Japanese non-valvular atrial fibrillation patients during anticoagulation therapy - warfarin vs direct oral anticoagulants. Circ J. 2017;81(5):645-51.

15. Zylla MM, Pohlmeier M, Hess A, Mereles D, Kieser M, Bruckner T, et al. Prevalence of intracardiac thrombi under phenprocoumon, direct oral anticoagulants (dabigatran and rivaroxaban), and bridging therapy in patients with atrial fibrillation and flutter. Am J Cardiol. 2015;115(5):635-40.

16 Di Minno MN, Ambrosino P, Dello Russo A, Casella M, Tremoli E, Tondo C. Prevalence of left atrial thrombus in patients with non-valvular atrial fibrillation. A systematic review and meta-analysis of the literature. Thromb Haemost. 2016;115(3):663-77.

17. Frenkel D, D'Amato SA, Al-Kazaz M, Markowitz SM, Liu CF, Thomas G, et al. Prevalence of left atrial thrombus detection by transesophageal echocardiography: a comparison of continuous non-vitamin $\mathrm{K}$ antagonist oral anticoagulant versus warfarin therapy in patients undergoing catheter ablation for atrial fibrillation. JACC Clin Electrophysiol. 2016;2(3):295-303.

18. Wyrembak J, Campbell KB, Steinberg BA, Bahnson TD, Daubert JP, Velazquez EJ, et al. Incidence and predictors of left atrial appendage thrombus in patients treated with nonvitamin $\mathrm{K}$ oral anticoagulants versus warfarin before catheter ablation for atrial fibrillation. Am J Cardiol. 2017;119(7):1017-22.

19. Lip GY, Hammerstingl C, Marin F, Cappato R, Meng IL, Kirsch $\mathrm{B}$, et al. Left atrial thrombus resolution in atrial fibrillation or flutter: results of a prospective study with rivaroxaban (X-TRA) and a retrospective observational registry providing baseline data (CLOT-AF). Am Heart J. 2016;178:126-34.

20. Kawakami T, Kobayakawa H, Ohno H, Tanaka N, Ishihara H. Resolution of left atrial appendage thrombus with apixaban. Thromb J. 2013;11(1):26.

21. Vaquerizo B, Sami M. Left atrial appendage thrombus resolution with reduced dose apixaban. J Atr Fibrillation. 2015;8(1):1182.

22. Vidal A, Vanerio G. Dabigatran and left atrial appendage thrombus. J Thromb Thrombolysis. 2012;34(4):545-7.

23. Marsico F, Cecere M, Parente A, Paolillo S, de Martino F, Dellegrottaglie $S$, et al. Effects of novel oral anticoagulants on left atrial and left atrial appendage thrombi: an appraisal. J Thromb Thrombolysis. 2017;43(2):139-48.

24. Mitamura H, Nagai T, Watanabe A, Takatsuki S, Okumura K. Left atrial thrombus formation and resolution during dabigatran therapy: a Japanese Heart Rhythm Society report. J Arrhythm. 2015;31(4):226-31.

25. Piotrowski R, Zaborska B, Pilichowska-Paszkiet E, Sikora-Frąc M, Baran J, Kułakowski P. RIVAroxaban TWICE daily for lysis of thrombus in the left atrial appendage in patients with nonvalvular atrial fibrillation: the RIVA-TWICE study. Arch Med Sci. 2020;16(2):289-96.

26. Watanabe T, Shinoda Y, Ikeoka K, Minamisaka T, Fukuoka H, Inui $\mathrm{H}$, et al. Dabigatran therapy resulting in the resolution of rivaroxaban-resistant left atrial appendage thrombi in patients with atrial fibrillation. Intern Med. 2017;56(15):1977-80. 
27. Kapłon-Cieślicka A, Budnik M, Gawałko M, Peller M, Gorczyca I, Michalska A, et al. Atrial fibrillation type and renal dysfunction as important predictors of left atrial thrombus. Heart. 2019;105(17):1310-5.

28. Zhou X, Wang Z, Dou S, Chen K, Liu E, Liu T, et al. Biomarkers for predicting left atrial or left atrial appendage thrombus in anticoagulated patients with nonvalvular atrial fibrillation. Cardiol Res Pract. 2020;2020:1683142.

29. Niku AD, Shiota T, Siegel RJ, Rader F. Prevalence and resolution of left atrial thrombus in patients with nonvalvular atrial fibrillation and flutter with oral anticoagulation. Am J Cardiol. 2019;123(1):63-8.

30. Hellwig T, Gulseth M. Pharmacokinetic and pharmacodynamic drug interactions with new oral anticoagulants: what do they mean for patients with atrial fibrillation? Ann Pharmacother. 2013;47(11):1478-87.

31. Ufer M. Comparative efficacy and safety of the novel oral anticoagulants dabigatran, rivaroxaban and apixaban in preclinical and clinical development. Thromb Haemost. 2010;103(3):572-85.

32. Lip GYH, Keshishian A, Li X, Hamilton M, Masseria C, Gupta K, et al. Effectiveness and safety of oral anticoagulants among nonvalvular atrial fibrillation patients. Stroke. 2018;49(12):2933-44.

Publisher's Note Springer Nature remains neutral with regard to jurisdictional claims in published maps and institutional affiliations. 